\title{
POLYMODEL DESCRIPTION AND QUALITATIVE ANALYSIS OF PROBLEMS FOR MEASUREMENT-COMPUTER OPERATIONS PLANNING IN CYBERPHYSICAL SYSTEMS
}

\author{
Boris Sokolov ${ }^{1}$, Oleg Tolpegin ${ }^{2}$, Yury Ipatov ${ }^{3}$, Yury Andrianov ${ }^{3}$ \\ ${ }^{1}$ St. Petersburg Institute for Informatics and Automation of the Russian Academy of Sciences (SPIIRAS), \\ Saint Petersburg, Russian Federation \\ ${ }^{2}$ Baltic State Technical University «Voenmeh» named after D.F. Ustinov, Saint Petersburg, \\ Russian Federation \\ ${ }^{3}$ Volga State University of Technology (Volgatech), Yoshkar-Ola, Russian Federation
}

The paper proposes a multimodal description of the cyberphysical systems functioning (CPS), which are multifunctional hardware and software systems intended for receiving (transmitting), storing, processing and forming control actions intended for both service objects (SO) performing a specified set of targets and not part of the CPS, and to ensure its own reliable operation. This description is based on the original dynamic interpretation of the corresponding processes. For concretization in the article, the planning stage of measurement-computing operations (MCO), conducted by the CPS both for determining its location and the position of the SO, is considered. The consideration of the MCO is caused by their special importance in solving the SO management problems. The paper shows how the problem of MCO planning can be achieved with the help of Pontryagin's maximum principle and reduce to a two-point boundary value problem. In this case, the task of planning the MCO is formulated as the task of finding the optimal program control, which ensures obtaining the required accuracy of the CPS location and SO for the minimum time (or with the minimum energy costs for the implementation of the MCO). Traditionally, tasks of this class (scheduling problems) are solved using mathematical programming methods. The proposed use of the optimal control theory methods for solving scheduling problems makes it possible to improve the quality of planning results (including increasing the efficiency of obtaining a plan, reducing the cost of resources when implementing it). Information on practical implementation of the developed models and algorithms is given.

Key words: Integrated scheduling; Dynamics models; Optimal program control;

\section{INTRODUCTION}

At present, knowledge is the main resource for accelerated development of the modern information society, the digital economy based on knowledge is the main development mechanism. The main technologies of the digital economy are new information and communication technologies, which are actually general-purpose technologies, like heat and power generation technologies. The main component of digital production and the digital economy as a whole are the various classes of cyberphysical systems (CPS), which include measuring, telecommunication and control subsystems [2].

CPSs are automatic control systems, by which existing and perspective natural and artificial objects of animate and inanimate nature can be combined by multi-channel (wired and wireless) measuring systems with built-in software into various types of hierarchical-network structures.

On the basis of the CPS, the projects of "smart production", "smart houses", "smart energy", "smart transport", "intelligent life safety system", "smart health", "safe smart cities", "smart" defense facilities etc. Such systems can ensure the implementation of controlled self-organization technologies in the organization of traffic on the streets of the city by analyzing the data coming from cars about their status and direction of traffic; coordinated functioning of production equipment for efficient production of small batches for various products, as well as electricity generation by optimizing the loading of thermal, nuclear and hydroelectric power plants, etc.

In this case, the measuring and computing subsystems of the CPS can be considered as variants of intelligent self-controlled measuring and computing systems that have a number of features. These features, in the first place, include the following [2]: the number of measuring channels in one CPS, even in the coming years can range from tens to hundreds of units; the measuring channels can include sensors of various magnitudes, both scalar and tensor, whereby the sensors can be spaced far apart; Measuring information is transmitted over long distances via wired or wireless channels; in the number of measured quantities there can be both scalar and tensor quantities. Processing of measurement information can be carried out using various computational technologies, including cloud computing, while the processing itself should be close to the real time scale.

Similar features are also characteristic for control subsystems of the CPS. These systems differ from previous built-in systems in the ability to plan and adapt their be 
havior according to environmental conditions and their expected (predictable) changes; learn new models and behavior scenarios and be self-managing, including those that implement the functions of proactive monitoring, management, and also metrological self-control. Their widespread introduction will lead to much greater changes than the emergence of computers and the Internet. The large-scale transition to CPS and related Internet of things is considered by many as the 4th industrial revolution, called "Industry 4.0"

Creation of cost-effective CPS is possible only if the data, information and knowledge on which they are based are highly reliable, arrive quickly, and operational costs are sufficiently small. An important role in ensuring the quality of functioning of the CPS is assigned to management processes, and among them to the planning of the functioning of these systems. The limited volume of the article does not allow us to dwell in detail on the merits and demerits of all existing approaches to solving the tasks for planning the work of the CPS.

Therefore, we will dwell only on the consideration of the question of compiling an operational plan for measuring and computing operations, as the most important and time-consuming stage of the integrated planning for the work of the CPS.

\section{A INFORMATIVE FORMULATION OF THE PLANNING TASK}

Let there be a number of service objects (SO): $\tilde{A}=\left\{\tilde{A}_{i j}, i \epsilon \tilde{N}\right\}$, forming part of some grouping $\mathrm{SO}$, solving together any specific target task (for example, monitoring the state of ecological and economic objects (EEO)). For the correct operation of the $\mathrm{SO}$, it is necessary to constantly update and correct the navigation information on board each SO. This task is performed by the CPS, which includes [2]: hardware and software systems that support the tasks of receiving (transmitting), storing, processing and generating control actions intended for both service objects (SO) that are not part of the CPS. and to ensure its own reliable operation). We introduce the set of CPS: $\widetilde{B}=\left\{\widetilde{B}_{j}, i \in \widetilde{M}\right\}, \widetilde{M}=\{1, \ldots, \widetilde{m}\}$. At the same time, due to the availability of unified hardware and software information communication tools on both the SO and the CPS, each of the listed elements of the SO and the CPS, with the availability of relevant information, is able to perform the functions of any other element in one or another extent depending on the emerging situation. Moreover, for the convenience of the following exposition, we introduce the aeneralized set of interacting objects $(\mathrm{IOb})$ $B=\left\{B_{l}, l, i, j \in M=\widetilde{N} \cup \widetilde{K} \cup \widetilde{M}=\{1, \ldots, m\}\right\}$. We also introduce one more set - the set of interaction operations (IOp) $D^{(i)}=\left\{D_{\gamma}^{(\mathrm{i})}, \gamma \in \Phi\right\}$.

In view of the foregoing, at the content level, the task for planning the work of the CPS for performing measurement and computational operations (which are a subset of SO) can be formulated as follows: it is necessary to find a permissible program for the management of the MCO and CPS (the plan of their operation), in the course of which implementation will be carried out in a timely manner and completely all the operations included in the corresponding technological control cycles (TCC) of the $\mathrm{SO}$, and the quality of the provision for SO with the given information would satisfy the given requirements.

At the same time, if several admissible control programs of the CPS are received, then it is necessary to choose the best (optimal) program (plan) in terms of accepted optimality criteria.

\section{DYNAMIC MODELS FOR PLANNING THE FUNCTIONING OF CPS}

The formalization of the planning task, as already indicated in the introduction, will be carried out using the dynamic interpretation of the process of performing technical operations proposed by the authors $[2,7,8]$. Based on the informative formulation of the task for planning the CPS, we introduce the following models of program management.

A dynamic model for managing interaction operations (including computational operations) in the CPS (Mo model) (1)

where $x_{i \gamma}^{(o)}$ - a variable that characterizes the state of execution IOb $D_{\gamma}^{(i)} ; a_{\gamma}^{(o)}, a_{i \tilde{\alpha}}^{(o)}, a_{i \widetilde{\beta}}^{(o)}$ - specified volumes of operations - control action, $u_{i v j}^{(o)}(t)=$ lif the operation $D_{\gamma}^{(i)}$ is performed, $u_{i \gamma j}^{(o)}(t)=0$ - otherwise; $\Gamma_{i y 1}, \Gamma_{i y 2}$ - the number of interaction interaction numbers carried out with the object $B_{i}$, immediately preceding and technologically related to the operation

$$
\begin{aligned}
& M_{o}=\left\{\mathbf{u}^{(o)}(t) \mid \dot{x}_{i \gamma}^{(o)}=\sum_{j=1}^{m} \varepsilon_{i j}(t) \cdot u_{i \gamma}^{(o)} ; x_{i \gamma}^{(o)}\left(t_{0}\right)=\mathrm{c}\right. \\
& x_{i \gamma}^{(o)}\left(t_{f}\right)=a_{i \gamma}^{(o)}, \sum_{i=1}^{m} \sum_{\gamma=1}^{s} u_{i \gamma \mathbf{j}}^{(o)} \leq c_{j}^{(o, 1)}, \\
& \sum_{j=1}^{m} \sum_{\gamma=1}^{s_{j}} u_{i \gamma \mathrm{j}}^{(0)} \leq c_{i}^{(0,2)}, u_{i \gamma \mathrm{j}}^{(0)}(t) \in\{0,1\}, \\
& u_{i \gamma \mathrm{j}}^{(o)}\left[\sum_{\tilde{\alpha} \in \Gamma_{i \gamma 1}}\left(a_{i \widetilde{\alpha}}^{(o)}-x_{i \widetilde{\alpha}}^{(o)}\right)+\prod_{\tilde{\beta} \in \Gamma_{i \gamma 2}}\left(a_{i \widetilde{\beta}}^{(o)}-x_{i \widetilde{\beta}}^{(o)}\right)\right]=0, \\
& \left.i, j=1, \ldots, m ; i \neq j ; \gamma=1, \ldots, s_{i}\right\},
\end{aligned}
$$


$D_{\gamma}^{(i)}$ with the help of logical operations "AND", "OR" respectively; $c_{i}^{(o, 1)}, c_{i}^{(o,}$ - given constants, characterizing the technical limitations associated with the functioning of the CPS as a whole; $\varepsilon_{i j}(t)$ - known matrix time function, with the help of which the space-time constraints associated with the interaction of objects $B_{i}\left(\right.$ or $\left.\bar{B}_{k}\right)$ with $B_{i}$, this function takes on a value 1 , if $B_{i}$ falls into a given interaction zone $B_{j ;}$ 0 - otherwise.

A dynamic model for controlling the parameters of measurement operations conducted in the CFS (model Me) (2)

$$
\begin{gathered}
M_{e}=\left\{\mathbf{u}^{(e)}(t) \mid \dot{\mathbf{x}}_{i}^{(g)}=F_{i}(t) \mathbf{x}_{i}^{(g)} ;\right. \\
y_{j}^{(i)}(t)=\mathbf{d}_{j}^{\mathrm{T}}(t) \mathbf{x}_{i}^{(g)}+\xi_{j}^{(e)} ; \\
\dot{Z}_{i}=-Z_{i} F_{i}-F_{i}^{\mathrm{T}} Z_{i}-\sum_{j=1}^{m} \sum_{\tilde{\gamma} \in \Gamma_{i}} u_{i \bar{\gamma} j}^{(e)} \frac{\mathbf{d}_{j} \mathbf{d}_{j}^{\mathrm{T}}}{\sigma_{j}^{2}} ; \\
\left.i \neq j ; i, j \in \tilde{M} ; 0 \leq u_{i \bar{\gamma} j}^{(e)} \leq c_{j \tilde{\gamma}}^{(e)} u_{i \gamma j}^{(o)}\right\},
\end{gathered}
$$

where $\mathbf{x}_{i}^{(g)}$ - state vector of SO $B_{i} ; F_{i}(t)$ - a given matrix characterizing the dynamics of changes in variables (calculated parameters) describing the state of the SO (for example, their spatial position); $\xi_{j}^{(e)}$ - uncorrelated errors in measurements of $\mathrm{SO}$ parameters carried out using technical means $B$ CPS; it is assumed that measurement errors obey the normal distribution law with zero mathematical expectation and a variance equal to $\sigma_{i}^{2} ; D_{\ddot{\gamma}}^{(i)} \in D^{(i)} ; u_{i \tilde{\gamma} j}^{(e)}(t)$ - control action, which determines the intensity of measurements $y_{j}^{(i)}(t) \mathrm{SO}$ (for example, range to $\mathrm{SO}$, temperature and humidity on board
SO), which are remotely conducted with the help of the technical means for CFS $B_{i} ; c_{i \tilde{y}}^{(e)}$ preset values characterizing the technical capabilities of the facilities $B$ during the operation $D_{\tilde{\gamma}}^{(i)} ; Z_{i}$ - matrix inverse to the correlation error matrix $K_{i}(t)$ of the estimation of the SO state vector $B_{i} ; \Gamma_{i}$ - a set of interaction operations conducted by the CPS with the SO $B_{i} ; d_{j}(t)$ - a given vector that determines the features of the technical implementation of the equation for the CPS meter $B_{i} ; K_{i 0}-K_{i}$ initial value $t=t_{0} ; \sigma_{y i}^{2}$ given accuracy of determination for $x$-th component of the SO state vector $\boldsymbol{x}_{i}^{(g)}(t)$ SO. The main difference between the model (2) and the proposed ones is that in it the operations of measuring the parameters of the SO state through constraints on the control actions $u_{i \bar{\gamma} j}^{(e)}$ are directly connected with the measurement and computation operations performed by the CPS specified in the model (1). This allows you to explore from a single system positions the task of planning $\mathrm{MCO}$ as a process of collecting data transmission and processing, and the task of planning measurements of parameters of monitored objects.

\section{Quality indicators for planning the functioning of the CPS}

Evaluation of the quality for the processes of the program management MCO CPS (or, in other words, the quality of the operational planning of the MCO) using the constructed dynamic models can be carried out, various objective functions $[4,5,11,12]$. For example, we give some of them:

$$
\begin{gathered}
J_{1}^{(o)}=\frac{1}{2} \sum_{i=1}^{m} \sum_{\gamma=1}^{s_{i}}\left\{\left[a_{i \gamma}^{(o)}-x_{i \gamma}^{(o)}\left(t_{f}\right)\right]^{2}+\sum_{j=1}^{m} \int_{t_{0}}^{t_{f}} \eta_{i \gamma}(\tau) u_{i \gamma j}^{(o)}(\tau) d \tau\right\}, i \neq j \\
J_{2}^{(e)}=\vec{b}_{\gamma}^{T} K_{i}\left(t_{f}\right) \vec{b}_{\gamma} ; J_{3}^{(e)}=\sum_{i=1}^{m} \sum_{j=1}^{m} \sum_{\gamma \in D^{(i)}} \int_{t_{0}}^{t_{f}} u_{i \not \gamma j}^{(e)}(\tau) d \tau, j \neq i
\end{gathered}
$$

where $\eta_{i y}(\tau)$ known monotonous time functions, which are selected taking into account the specified directive dates for the beginning (completion) of the $\mathrm{IOb} \mathrm{SO} \mathrm{im}$ plementation with the CFS $B_{i}$. Indicator (3) is introduced if it is necessary to evaluate the completeness of the implementation for the boundary conditions, as well as the amount of the total penalty for failure to meet the specified deadlines for performing operations. For SO that solve the tasks of monitoring the state of the EEO, the operations related to assessing their locations, which in turn will determine the location of the EEO, are of particular importance. In this regard, the magnitude of the quality index $J_{3}^{(e)}$ characterizes the accuracv of $x$-th determination of the vector component $x_{i}^{(g)} \quad\left(\vec{b}_{\chi}=\|00 \ldots 1 \ldots 00\|^{T}\right.$ - a given auxiliary vector that separates in the correlation matrix $K_{i}(t)$ required item number $x$ ). The objective function $J_{4}^{(e)}$ allows you to quantify the total costs of CPS re- sources when performing operations $D_{\tilde{\gamma}}^{(i)}$ related to the measurement of the $\mathrm{SO}$ status.

\section{The formal formulation of the problem for planning the measurement and computing operations performed by the CPS}

On the basis of the above partial dynamic models, we will write down the generalized dynamic model for the processes of interaction between the CPS with SO (5). Where $\quad \vec{x}=\left\|\vec{x}^{(o) \mathrm{r}} \vec{x}^{(e) \mathrm{r}}\right\|^{\mathrm{T}} ; \vec{u}=\left\|\vec{u}^{(o) \mathrm{r}} \mathrm{u}^{(e) \mathrm{T}}\right\|^{\mathrm{T}}$ - generalized vectors of state and control of CPS, as well as SO, the components of which are the corresponding variables included in the model Mo, Me; $\vec{h}_{0}, \vec{h}_{1}$ - known vector-valued functions, with the aid of which boundary conditions for the vector $\vec{x}$ are given at time instants $t=t_{0}$ and $t=t_{p}$ $\vec{q}^{(1)}, \vec{q}^{(2)}$ - vector functions, with the help of which the

$$
M=\left\{\vec{u}(t) \mid \dot{\vec{x}}=\vec{f}(\vec{x}, \vec{u}, t) ; \vec{h}_{0}\left(\vec{x}\left(t_{0}\right)\right) \leq \vec{O} ; \vec{h}_{1}\left(\vec{x}\left(t_{f}\right)\right) \leq \vec{O}, \vec{q}^{(1)}(\vec{x}, \vec{u})=\vec{O} ; \vec{q}^{(2)}(\vec{x}, \vec{u}) \leq \vec{O}\right\}
$$


basic space-time, technical and technological constraints imposed on the process of functioning for the CPS and SO are specified.

In addition to expression (5), the deterministic dynamic model of MCO planning should include the system of planning quality indicators, given by the following generalized vector index:

$$
\vec{J}_{o \sigma}=\left\|J_{1}^{(o)} J_{2}^{(e)} J_{3}^{(e)}\right\|^{\mathrm{T}}
$$

components of which are partial indicators of the quality planning (3)-(4).

In this case, the planning task can be formulated as the next task of programmatic control of the dynamic system (1)-(2): it is necessary to find such admissible control $\vec{u}(t), t \epsilon\left(t_{0}, t_{f}\right]$, which satisfies the required restrictions $\vec{q}^{(1)}(\vec{x}, \vec{u})=\vec{O}, \vec{q}^{(2)}(\vec{x}, \vec{u}) \leq \vec{O}$ and translates the dynamic system $\dot{\vec{x}}=\vec{f}(\vec{x}, \vec{u}, t)$ from the given initial state $\vec{h}_{0}$ in a given final state $\vec{h}_{1}$. If there are several such control actions (plans), then among them the best (optimal) is chosen, at which the components of the generalized vector (6) take extreme values. In carrying out a qualitative analysis of the problem posed, it was assumed that the scalar vector of the planning quality by form (6) [7].

\section{QUALITATIVE ANALYSIS OF THE TASK PLANNING THE FUNCTIONING FOR THE CPS}

The formulation of the planning task as a control problem for a dynamical system of the form (1) - (2) allows, with its qualitative analysis, to widely use the mathematical theory of control theory $[1,4,11,12,14]$. Due to the limited volume of the article, we will only discuss some aspects of the qualitative analysis for the planning problem. First of all, let us analyze the control object itself of the form (1) - (2). It is described as a deterministic non-stationary

linear finite-dimensional differential dynamical system of large dimension, whose right-hand parts fail not only at the moments of switching control actions, but also at times corresponding to the entrance time (exit) of the SO into the interaction zones with the CPS. In contrast to the previously proposed approaches [6,9,11-13], all the basic spatio-temporal, technical and technological constraints in models (1) - (2), which have a nonlinear character, are taken into account not for specifying differential equations, but for when forming the area of permissible control actions $Q(\vec{x}(t))$, and also setting boundary conditions. This feature of the description made it possible to obtain the following interesting result when analyzing the existence of an optimal solution in the stated planning problem.

In addition to the initial class of admissible control actions $\widetilde{K}_{\sigma}$, given by constraints $\vec{q}^{(1)}$ and $\vec{q}^{(2)}$ describing the region $Q(\vec{x}(t))$, it was pronosed to consider an extended class of control actions $\widetilde{K}_{\sigma}^{(p)}$, in which the conditions for the relay control of controls of the form $u_{i \gamma j \lambda}^{(o)}(t) \in\{0,1\}$ are replaced by conditions $u_{i \gamma j \lambda}^{(o)}(t) \in[0,1]$ (a transition from $\vec{u}$ to $\vec{u}_{p}$ is made).

In this case, using special transformations, it is possible to form an extended area of admissible controls $O(\vec{x}(t))$ in such a way that it is convex and compact and satisfies the conditions of general position $[1,4,7,8]$. It follows from the analysis of the work that for an extended class of admissible controls $\widetilde{K}^{(p)}$ all the conditions of the existence theorem for optimal control in problems of this class are satisfied [4].

In this case, if there is an optimal control $\vec{u}_{p}^{*}$ in the class $\widetilde{K}_{\sigma}^{(p)}$, then, as follows from the method of local sections, this control at each instant of time $t \epsilon\left(t_{0}, t_{f}\right]$ (with the exception of the points of discontinuity) delivers a maximum on the set $Q_{p}(\vec{x}(t))$ to the Hamiltonian (7)

$$
H\left(\vec{x}^{*}(t), \vec{u}^{*}(t), \vec{\psi}^{*}(t)\right)=\max _{\vec{u}_{p} \in Q_{p}(\vec{x})} \sum_{\tilde{p}=1}^{3} H_{\tilde{p}}(\vec{x}(t), \vec{u}(t), \vec{\psi}(t))
$$

where $\mathrm{H}_{1}=\sum_{i=1}^{m} \sum_{\gamma=1}^{s_{t}} \sum_{\substack{j=1 \\ i \neq j}}^{m}\left(\psi_{i \gamma}^{(o)} \varepsilon_{i j}+\eta_{i \gamma}(\tau)\right) u_{i \gamma j}^{(o)} \quad \mathrm{H}_{2}=\sum_{i=1}^{m} \sum_{\substack{j=1 \\ i \neq j}}^{m} \sum_{\gamma=1}^{s_{t}} \sum_{\rho=1}^{\lambda_{i}}\left(\psi_{i \gamma j \rho}^{(f, 1)} \cdot u_{i \gamma j \rho}^{(f, 1)}+\psi_{i \gamma j \rho}^{(f, 2)} \cdot u_{i \gamma j \rho}^{(f, 2)}\right)$

$$
\mathrm{H}_{3}=-\Psi_{i}^{(e) T} * Z_{i} F_{i}-\Psi_{i}^{(e)} * F_{i} Z_{i}+\sum_{j=1}^{m}\left(\psi_{i}^{(e) T} * V_{o j}-1\right) u_{i \tilde{\gamma} j}^{(e)} \text { here with } V_{o j}=\frac{1}{\sigma_{j}^{2}} \vec{d}_{j} \vec{d}_{j}^{T} \quad \text { sign * }
$$

denotes the operation of the product for the corresponding Hadamard matrices. In this case, a continuous solution of the auxiliary conjugate system can be found from the following system of differential equations $[1,3,8](8)$. Satisfying the transversality conditions on the left and right end of the phase trajectory $\vec{x}(t)$ of the dynamical

$$
\begin{gathered}
\dot{\psi}_{l}=-\frac{\partial H}{\partial x_{l}}+\sum_{\alpha=1}^{I_{l}} \lambda_{\alpha}(t) \frac{\partial q_{\alpha}^{(1)}(\vec{x}(t), \vec{u}(t))}{\partial x_{l}}+\sum_{\beta=1}^{I_{2}} \rho_{\beta}(t) \frac{\partial q_{\beta}^{(2)}(\vec{x}(t), \vec{u}(t))}{\partial x_{l}}, l=1, \ldots, \widetilde{n} \\
\rho_{\beta}(t) q_{\beta}^{(2)}(\vec{x}(t), \vec{u}(t)) \equiv 0, \beta \in 1, \ldots, I_{2} \\
\operatorname{grad}_{\vec{u}} H(\vec{x}(t), \vec{u}(t), \vec{\psi}(t))=\sum_{\alpha=1}^{I_{1}} \lambda_{\alpha}(t) \operatorname{grad}_{\vec{u}} q_{\alpha}^{(1)}(\vec{x}(t), \vec{u}(t))+\sum_{\beta=1}^{I_{2}} \rho_{\beta}(t) \operatorname{grad}_{\vec{u}} q_{\beta}^{(2)}(\vec{x}(t), \vec{u}(t))
\end{gathered}
$$


system (5).

The coefficients and are found from the following relations (9) and (10).

In the formulas (9)-(10) $x_{i}, \psi_{i}$ - components of generalized state vectors $\vec{x}$ and conjugate system of equations $\vec{\psi} ; q_{\alpha}^{(1)}(\vec{x}(t), \vec{u}(t)), q_{\beta}^{(2)}(\vec{x}(t), \vec{u}(t))$ - components of the original constraint system included in the expression (7). In $[3,7,8]$ examples of the specification of conjugate systems of equations for various classes of applied problems are given.

The analysis of expression (7) shows that the Hamiltonian is linear by $\vec{u}_{p}$, so its maximum is attained at one of the extreme points for the set $Q_{p}(\vec{x}(t))$, and since $Q_{p}(\vec{x}(t))$ is a linear hull $Q(\vec{x}(t))$, the set $Q(\vec{x}(t))$ is the union of the indicated extreme points. Hence it follows that the maximization of the Hamiltonian (7) on sets $Q$ and $Q_{p}$ leads to the same result. Therefore, the optimal control in the class $\widetilde{K}_{\sigma}^{(p)}$ is an element of the class $\widetilde{K}_{\sigma}$ and, since $\widetilde{K}, \tau \widetilde{K}_{\sigma}^{(p)}$, then it is also optimal with respect to the class $\widetilde{K}_{\sigma}$. Thus, when solvinn the relaxed problem of MCO planning in a class $\widetilde{K}_{\sigma}^{(p)}$, solutions are obtained (the nlan) admissible in the initial class of control actions $\widetilde{K}_{\sigma}^{(p)}$. In $[1,4,10]$, for problems of this class, it was proved that the formulated necessary conditions for optimality are simultaneously sufficient conditions.

\section{CONCLUSION}

The paper contains a poly-model description and a qualitative analysis of the tasks for the complex planning $\mathrm{MCO}$ by CPS. The main features and differences of the proposed models are that when dynamically interpreting the processes of performing the MCO entering the technological cycle (TC) of the CPS, the dimension of the solved planning tasks and the degree of connectivity for the scheduling algorithm are significantly reduced. This dimension is determined when solving the problem of planning at each time point by the number of independent paths in the general network diagram of the work performed by the CPS, the current spatio-temporal, technical, and technological constraints. At the same time, in each such time section several small-size assignment problems and / or linear programming are solved (see models (1), (2)). It should be noted that in this case the variables and constraints that were relevant up to the moment or will be relevant after, do not influence the current dimension of the tasks of resource allocation of the CPS. In turn, the degree of connectivity for the scheduling algorithm is determined by the dimensionality of the state vectors of the basic and coniugate systems equations: $\vec{x}\left(t^{\prime}\right), \vec{\psi}\left(t^{\prime}\right), t^{\prime} \in\left(t_{0}, t_{f}\right], t^{\prime}$ the time at which the planning task was interrupted. Remembering the value of these vectors, you can resume the calculation of the work plan of the CFC as soon as the relevant restrictions are removed.

Studies of the properties and characteristics of the developed models have shown that, due to rational (optimal) planning of the operation of the CPS, firstly, the capacity of the CPS as a whole is increased, secondly, the lags in the control circuits of the CPS are reduced, and thirdly, loads with sudden changes in the structure of the CPS. In addition, based on the dynamic description of the functioning for the CPS, it is possible to explicitly associate the control technology with its elements and subsystems with the results of the targeted application of the SO, which accept data processing and analysis, as well as with the characteristics of the hardware and software complexes for the CPS. Thus, at the same time MCO planning opens up interesting prospects for the formation of justified requirements for the characteristics of the HSC CPS, for example, to the parameters of the navigation field created by them. The site http://litsam.ru provides information on numerous practical implementations of the proposed approach to solving the problems of scheduling theory that arise in different subject areas (astronautics, shipbuilding, public administration, etc.).

\section{RESEARCH SUPPORT}

The results of the research were carried out with the financial support of the Ministry of Education and Science of the Russian Federation №2.3135.2017/4.6 (section 1-4,6), by state research №0073-2018-000 (section 5).

\section{REFERENCES}

1. Boltyanskij V.G., (1969). Mathematical methods of optimal control. Moscow: Nauka.

2. Kupriyanovskij V.P., Namiot D.E., \& Sinyagov S.A., (2016). Cyber-physical systems as a base for digital economy. International Journal of Open Information Technologies, 4(2), 18-24.

3. Kalinin V.N., \& Sokolov B.V., (1987). Dinamicheskaya model' i algoritm optimal'nogo planirovaniya kompleksa rabot s zapretami na preryvanie. Automation and Remote Control, 1, 106-114.

4. Lee E.B., \& Marcus L., (1972). Foundations of Optimal Control Theory. Moscow: Nauka.

5. Lyubushin, A.A. (1979). Modifications and study of convergence of the method of successive approximations for optimal control problems. Zh. vychisl Mkt. mar. Fiz., 19(6), 1414-1421.

6. Malyshev V.V., Krasil'shchikov M.N., , \& Karlov V.I., (1989). Optimization of observation and control of flying vehicles. Moscow: Mashinostroenie.

7. Okhtilev M. Yu., Sokolov B.V., \& Yusupov R.M., (2006). Intellectual technologies for monitoring the state and managing the structural dynamics of complex technical objects. Moscow: Nauka.

8. Sokolov B.V., \& Yusupov R.M., (2002). Kompleksnoe modelirovanie funkcionirovaniya avtomatizirovannoj sistemy upravleniya navigacionnymi kosmicheskimi apparatami. Problemy upravleniya i informatiki, 5, 103-117.

9. Tanaev, B.C., \& Shkurba V.B., (1975). Introduction to 
scheduling theory. Moscow: Nauka.

10. Chernous'ko F.L, \& Kolmanovskii V.B., (1978). Optimal Control under Random Disturbances. Moscow: Nauka.

11. Ackoff R.L., (1978). The Art of Problem Solving. New York: Wiley-Interscience.
12. Gonzalez T., \& Sahni S., (1978). Flow Shop and Job Shop Scheduling: Complexity and Approximation. Operations Research, 26, 36-52.

13. Preuzeto sa http://litsam.ru.

14. Wolf W., (2009). Cyber-physical systems. Computer, $3,88-89$. 